\title{
Helicoidal dynamics of biaxial curved rods in twist-bend nematic phases unveiled by unsupervised machine learning techniques
}

DOI:

10.1103/PhysRevE.102.040601

\section{Document Version}

Accepted author manuscript

Link to publication record in Manchester Research Explorer

Citation for published version (APA):

Chiappini, M., Patti, A., \& Dijkstra, M. (2020). Helicoidal dynamics of biaxial curved rods in twist-bend nematic phases unveiled by unsupervised machine learning techniques. Physical Review E, 102(4).

https://doi.org/10.1103/PhysRevE.102.040601

\section{Published in:}

Physical Review E

\section{Citing this paper}

Please note that where the full-text provided on Manchester Research Explorer is the Author Accepted Manuscript or Proof version this may differ from the final Published version. If citing, it is advised that you check and use the publisher's definitive version.

\section{General rights}

Copyright and moral rights for the publications made accessible in the Research Explorer are retained by the authors and/or other copyright owners and it is a condition of accessing publications that users recognise and abide by the legal requirements associated with these rights.

\section{Takedown policy}

If you believe that this document breaches copyright please refer to the University of Manchester's Takedown Procedures [http://man.ac.uk/04Y6Bo] or contact uml.scholarlycommunications@manchester.ac.uk providing relevant details, so we can investigate your claim.

\section{OPEN ACCESS}




\title{
Helicoidal dynamics of biaxial curved rods in twist-bend nematic phases unveiled by unsupervised machine learning techniques
}

\author{
Massimiliano Chiappini, ${ }^{1, *}$ Alessandro Patti, ${ }^{2}$ and Marjolein Dijkstra ${ }^{1, \dagger}$ \\ ${ }^{1}$ Soft Condensed Matter, Debye Institute for Nanomaterials Science, Department of Physics, \\ Utrecht University, Princetonplein 1, Utrecht 3584 CC, The Netherlands \\ ${ }^{2}$ Department of Chemical Engineering and Analytical Science, \\ The University of Manchester, Manchester, M13 9PL, UK
}

(Dated: September 7, 2020)

\begin{abstract}
Uniaxial rods in a nematic phase diffuse preferentially in the direction parallel to the nematic director $\hat{\mathbf{n}}$. The nematic director field $\hat{\mathbf{n}}(\mathbf{r})$ of a chiral twist-bend nematic $\left(N_{T B}\right)$ phase of achiral banana-shaped particles, recently discovered experimentally, displays a heliconical twist of given handedness and periodicity. Using simulations, we investigate the long-time macroscopic diffusion in $N_{T B}$ phases, and find that the predilection of curved rods to diffuse in the direction of the twisting $\hat{\mathbf{n}}(\mathbf{r})$ yields a fascinating chiral dynamics along helices even though achiral curved rods display Brownian motion with a non-trivial roto-translational coupling. We devise a machine learning protocol to characterize the helicoidal particle trajectories, finding that their pitch and radius are determined by the pitch and conical angle of the $N_{T B}$ phase, thereby connecting its structural and dynamical properties.
\end{abstract}

Liquid crystals are phases of matter that combine properties from both solid and liquid phases. They may flow like liquids, but display degrees of orientational and/or translational order that resemble those of crystalline solids. In nematic liquid crystals the positions of molecules are random, but their axes are on average oriented along a common nematic director $\hat{\mathbf{n}}$. Due to the ease to manipulate this director with electric fields, nematic liquid crystals have revolutionized the way we display information with devices ranging from organic LEDs to LCDs in smart phones, laptop screens, and flatpanel TVs [1-5]. Very recently, a fascinating chiral nematic phase has been discovered in which the orientation of achiral banana-shaped bent-core mesogens twists and bends at a very small length scale [6-18]. This twist-bend nematic $\left(N_{T B}\right)$ phase is characterised by an oblique heliconical winding of the nematic director field $\hat{\mathbf{n}}(z)=\sin \left(\theta_{0}\right) \cos (q z) \hat{\mathbf{e}}_{x}+\sin \left(\theta_{0}\right) \sin (q z) \hat{\mathbf{e}}_{y}+\cos \left(\theta_{0}\right) \hat{\mathbf{e}}_{z}$, with $q=2 \pi / p$ the wave number of the heliconical twist, $\theta_{0}$ the conical angle, and $p$ the pitch length (see FIG. 1 (b)) $[19,20]$. The fluid-like behavior as well as the short length scale of the periodic modulations of the director makes this phase sensitive to external fields and highly promising for ultra-fast optoelectronic applications. Understanding the effect of the macroscopic chiral symmetry breaking on the transport properties of the $N_{T B}$ phase is hence not only interesting from a fundamental point of view, but also crucial for future technological applications.

To study the dynamics in an $N_{T B}$ phase one requires a model system that stabilizes this peculiar liquid crystal phase. Despite numerous efforts, simulations that succeeded to stabilize an $N_{T B}$ phase are scarce [21-23]. We

\footnotetext{
*m.chiappini@uu.nl

$\dagger$ m.dijkstra@uu.nl
}

recently discovered that an $N_{T B}$ phase can be stabilized in systems of curved hard rods [18]. In this Letter, we use this model to study the long-time translational diffusion in $N_{T B}$ phases of various handedness, pitch, and conical angle, and compare it with the macroscopic transport properties of isotropic and nematic phases.

To this end, we require a simulation method that mimics Brownian dynamics. In the limit of very small displacements, Monte Carlo (MC) methods have been shown to correctly mimic Brownian motion [24-28]. These Dynamic Monte Carlo (DMC) methods have been mostly applied to uniaxial particles with an infinite-fold rotational axis, without any coupling between translational and rotational motion. We notice that DMC has also been applied to investigate the dynamics of colloidal cuboids, but such a roto-translational coupling was neglected [29]. Here, we devise a DMC method for biaxial particles that display Brownian motion with a nontrivial roto-translational coupling at infinite dilution. Despite being valid for particles of arbitrary shape, we will discuss the method for our curved rods for simplicity. We consider a curved rod with position and orientation described by a six-dimensional vector $\mathbf{x}=(\mathbf{r}, \omega)=(x, y, z, \alpha, \beta, \gamma)$, where $x, y$, and $z$ are the center-of-mass coordinates with respect to a fixed laboratory reference frame, and $\alpha, \beta$, and $\gamma$ the Euler angles. Brownian motion is characterized by correlations between translational and/or rotational displacements at infinite dilution linear in time with the $6 \times 6$ real symmetric diffusion tensor $\mathbf{D}_{0}$, i.e. $\left\langle\Delta \mathbf{x}(t) \Delta \mathbf{x}^{T}(t)\right\rangle=2 \mathbf{D}_{0} t$. For uniaxial particles, $\mathbf{D}_{0}=$ $\operatorname{diag}\left(D_{0,1}, \cdots, D_{0,6}\right)$ is diagonal if the rotation axis is chosen along one of the axes of the molecular frame. In the DMC integration scheme of Ref. [24], a trial move $\mathbf{x}^{\mathbf{o}} \rightarrow \mathbf{x}^{\mathbf{n}}=\mathbf{x}^{\mathbf{o}}+\boldsymbol{\delta}$ simultaneously modifies all degrees of freedom of a randomly selected particle, with $\mathbf{x}^{\mathbf{n}}$ and $\mathbf{x}^{\mathbf{o}}$ denoting the new and old configurations, respectively, and $\delta$ the particle displacement. The $i$ th component of $\boldsymbol{\delta}$ is uniformly sampled within the interval $\left[-\Delta x_{i}, \Delta x_{i}\right]$, 

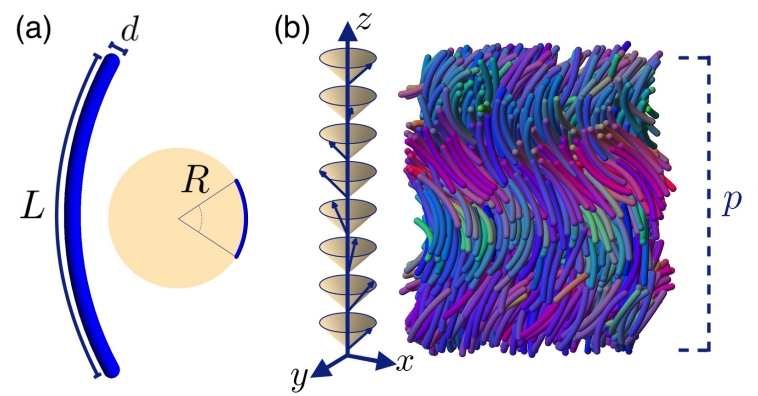

(c)

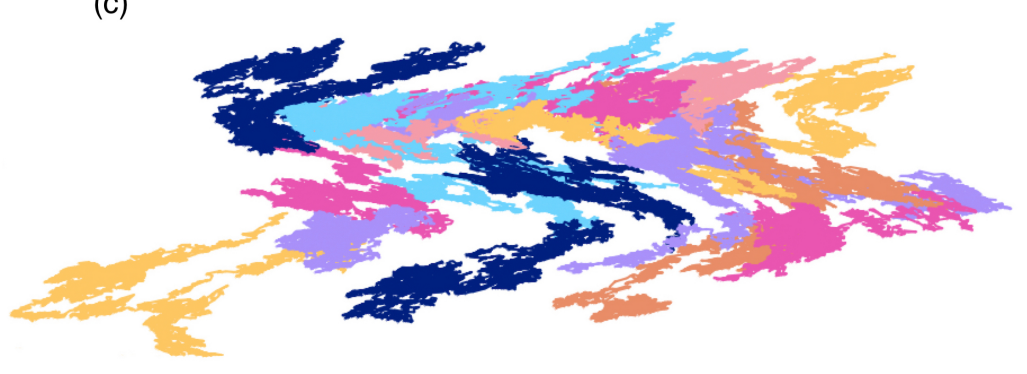

FIG. 1. (a) Sketch of a hard curved spherocylinder (HCS) of diameter $d$, length $L$ and radius of curvature $R$. (b) Typical simulation configuration of a right-handed $N_{T B}$ phase of HCSs with a sketch of the heliconical precession of the nematic director field. (c) Typical trajectories of HCSs in an $N_{T B}$ phase from DMC simulations, showing a peculiar helicoidal Brownian dynamics. Different colors denote different particle trajectories.

and $\Delta x_{i}$ is tuned according to the $i$ th diagonal element of $\mathbf{D}_{0}$ as $\Delta x_{i}=\sqrt{2 D_{0, i} \delta t_{M C}}$, where $\delta t_{M C}$ is the $\mathrm{MC}$ time step [30]. As a result, an MC cycle can be related to the Brownian dynamics time scale [24]. To generalize this method to biaxial particles with a roto-translational coupling, as our curved rods, we first diagonalize the diffusion tensor at infinite dilution $\mathbf{D}_{0}$ via $\mathbf{D}_{0}^{\prime}=\mathbf{Q}^{T} \mathbf{D}_{0} \mathbf{Q}$, with $\mathbf{Q}^{T}$ a change of basis matrix. In this new basis, $\mathbf{D}_{0}^{\prime}=\operatorname{diag}\left(D_{0,1}^{\prime}, \cdots, D_{0,6}^{\prime}\right)$ is diagonal, and we can apply the DMC scheme attempting a displacement $\boldsymbol{\delta}^{\prime}$ whose $i$ th component $\delta_{i}^{\prime}$ is uniformly sampled within the interval $\left[-\Delta x_{i}^{\prime}, \Delta x_{i}^{\prime}\right]$, with $\Delta x_{i}^{\prime}=\sqrt{2 D_{0, i}^{\prime} \delta t_{M C}}$. Transforming $\boldsymbol{\delta}^{\prime}$ back to the original basis, we obtain the trial move $\mathbf{x}^{\mathbf{o}} \rightarrow \mathbf{x}^{\mathbf{n}}=\mathbf{x}^{\mathbf{o}}+\mathbf{Q} \delta^{\prime}$. This integration scheme correctly mimics the Brownian motion of particles with rototranslational coupling in their diffusion [30]. In particular, the roto-translational coupling is expected to play a role at sufficiently low density, and should be less relevant in denser states, where the diffusion of single particles is mainly determined by crowding and macroscopic order. We also expect the roto-translational coupling to be relevant in the phase switching dynamics of exotic biaxial particles and the associated response time, e.g. switching from uniaxial to biaxial [31] or twist- and splay-bend [32] nematic phases upon application of an external field.

We study the dynamics of hard curved spherocylinders (HCSs) with diameter $d$, length $L=10 d$, and radius of curvature $R=9.5 d$, see FIG. 1 (a). Each HCS is modelled as a rigid chain of $N_{s}=L / d$ equally spaced spheres of diameter $d$. We use standard NPT-MC simulations to equilibrate systems of $N_{\mathrm{HCS}}=2048 \mathrm{HCSs}$ at varying packing fractions $\eta=N_{\mathrm{HCS}} v_{0} / V$ in the isotropic $(I)$, uniaxial nematic $(N)$, and $N_{T B}$ phases, with $v_{0}$ and $V$ the particle and box volume, respectively. The phase behavior of this system exhibits an $I-N$ transition at $\eta_{I N}=0.37 \pm 0.01$ and an $N-N_{T B}$ transition at $\eta_{N N_{T B}}=0.43 \pm 0.01$ [18]. The global nematic director is taken to be along the $z$ axis of the lab frame. The diffusion tensor of HCSs at infinite dilution $\mathbf{D}_{0}$ - calculated via the open-source software Hydro++ [33] — is non-

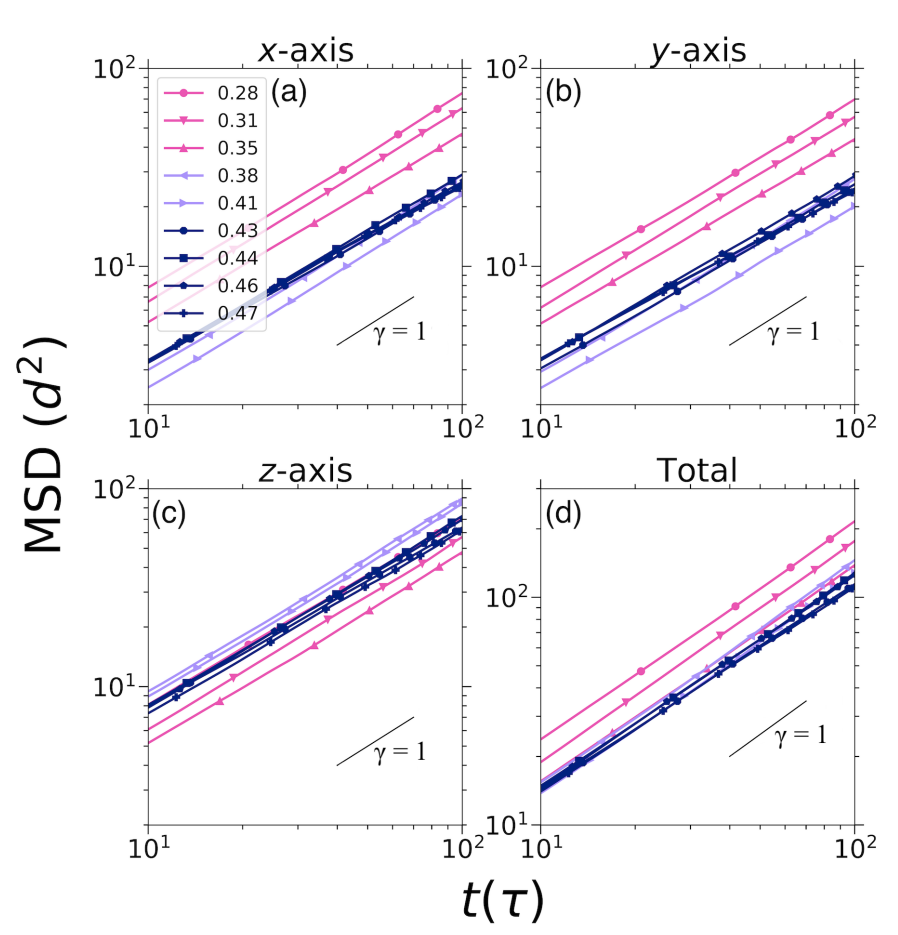

FIG. 2. Mean square displacements (MSDs) of HCSs with length $L=10 d$ and curvature $R=9.5 d$ along the (a) $x$, (b) $y$, (c) $z$ axes, and (d) total MSD for various packing fractions $\eta$ as reported in the legend. All MSDs show a long-time diffusive behavior $\left(\operatorname{MSD}_{\alpha}(t) \sim D_{\alpha} t^{\gamma}\right.$ with $\gamma=1$, for $\left.\alpha=x, y, z\right)$ of HCSs. The slope $\gamma=1$ corresponding to diffusive behavior is shown as a reference.

diagonal, implying that HCSs exhibit a roto-translational coupling [34-38]. In particular, their main mode of diffusion is a translation along the long particle axis coupled with a rotation [30]. In order to investigate how the roto-translational coupling affects the macroscopic transport in the various phases, we employ $\mathbf{D}_{0}$ in our novel DMC method and measure the mean square displacements $\mathrm{MSD}_{\alpha}$ along the $\alpha$-axis, with $\alpha=x, y, z$ (see 


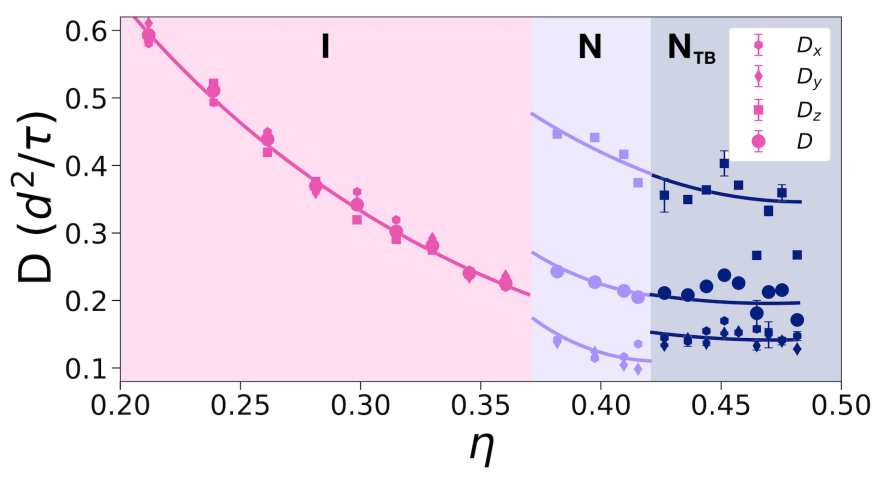

FIG. 3. Diffusion coefficients $D_{\alpha}$ with $\alpha=x, y, z$ and total diffusion coefficient $D$ of HCSs with length $L=10 d$ and curvature $R=9.5 d$ as a function of $\eta$. In the $N$ phase the dynamics is anisotropic with a faster diffusion along the nematic director. The anisotropy persists in the $N_{T B}$ phase, although weakened by an oblique helicoidal nematic director field [30]. The lines are guides to the eye. Some error bars are smaller than the symbols and thus not visible.
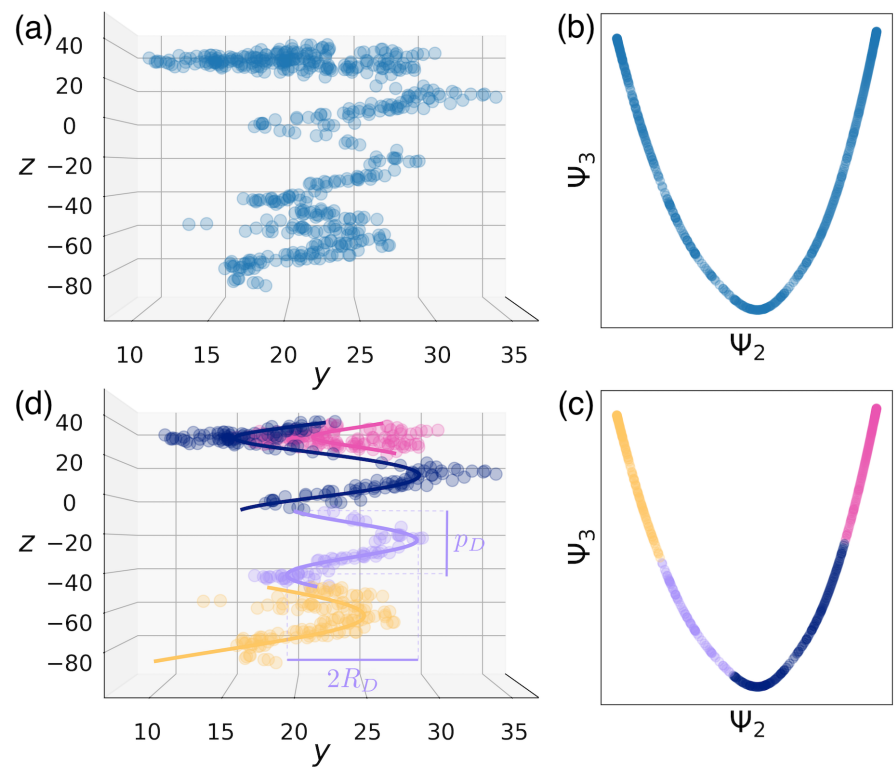

FIG. 4. Exemplary clustering and fit of an helicoidal trajectory via the Diffusion Maps (DMs) and the Hierarchical Agglomerative Clustering (HAC) method. The trajectory $\left\{\mathbf{r}_{i}=\left(x_{i}, y_{i}, z_{i}\right)\right\}$ in the 3D Euclidean space (a) is used as input for DMs, which yields a true dimensionality of the trajectory between 1 and 2 . The two principal components resulting from the DMs analysis are used to reparameterize the trajectory as $\left\{\mathbf{r}_{i} \rightarrow \boldsymbol{\Xi}_{i}=\left(\Psi_{2, i}, \Psi_{3, i}\right)\right\}$, obtaining a $2 \mathrm{D}$ trajectory (b) to which HAC is applied, resulting in clusters of points as denoted by different colors (c). The same clustering is transported to the original 3D trajectory (d), where different clusters can be fitted with the equation of a helix of radius $R_{D}$ and pitch $p_{D}$. These fits are shown as lines denoted by the same color as the fitted cluster.
FIG. 2), and the long-time translational diffusion coefficients $D_{\alpha}$ as $\operatorname{MSD}_{\alpha}(t) \sim D_{\alpha} t$ in equilibrated systems at fixed $\eta$ (see FIG. 3). In the $I$ phase, the diffusion coefficients are equal within our statistical accuracy in all directions. Upon increasing the density, the overall dynamics gets slower and the diffusion coefficients decrease. We observe a drastic change at the $I-N$ phase transition, where the system becomes anisotropic. The diffusion in the direction of the nematic director becomes faster than perpendicular to it, i.e. $D_{z}>D_{x y}$ with $D_{x y}=\left(D_{x}+D_{y}\right) / 2$, in agreement with previous works $[39,40]$. The anisotropy of the diffusion, $D_{z} / D_{x y}$, is weakened at the $N-N_{T B}$ transition [30] as the tendency of curved rods to diffuse along the local nematic director leads to a preferred direction of motion which is oblique in the $N_{T B}$ phase as $\hat{\mathbf{n}}(z)$ forms an angle $\theta_{0}$ with the $z$-axis.

Even though the main mode of Brownian diffusion of achiral curved rods is a coupled translation and rotation, we find that they display a fascinating chiral helicoidal dynamics with the same handedness as the host phase as evident from the exemplary center-of-mass trajectories of HCSs in an $N_{T B}$ phase shown in FIG. 1 (c), which is due to the predilection of curved rods to diffuse in the direction of the twisting nematic director field. Quantitatively assessing the properties of this dynamics is challenging. Most of the quantities usually employed to analyze transport properties like MSDs, diffusion coefficients, Van Hove functions, average out the unique features of this single-particle dynamics. One might be tempted to fit the trajectories with the equation of a helix to obtain an estimate of the radius $R_{D}$ and pitch $p_{D}$ of the chiral diffusion, but the diffusive dynamics in the $x y$ plane suggests that the curved rods are not constrained to diffuse along a single helicoidal trajectory. This naive picture would indeed result into a cage-trapping plateau in the transverse MSDs at $\sim R_{D}^{2}$, as the $x$ and $y$ positions would be constrained within the range $\left[-R_{D}, R_{D}\right]$. More importantly, the ergodicity hypothesis would be violated if the particle dynamics was restricted to a single helicoidal path. This suggests a more involved scenario in which each curved rod diffuses along different helices at different times, each with its own center and perhaps even with its own pitch and radius. Identifying the various helicoidal parts of a particle trajectory is therefore fundamental.

A simple cluster analysis of the raw particle trajectories would fail because of the non-linearity of the helicoidal paths - different points might be far in Euclidean space and yet belong to the same helix. The nonlinearity of the dynamics also makes linear dimensionality reduction methods such as a Principal Component Analysis (PCA), which would successfully pick up the anisotropy of the diffusion in a uniaxial $N$ phase, ineffective. Recently, non-linear dimensionality reduction algorithms have been successfully applied to characterize the low-dimensional dynamics of high-dimensional systems [41]. For example, the Diffusion Maps (DMs) method 

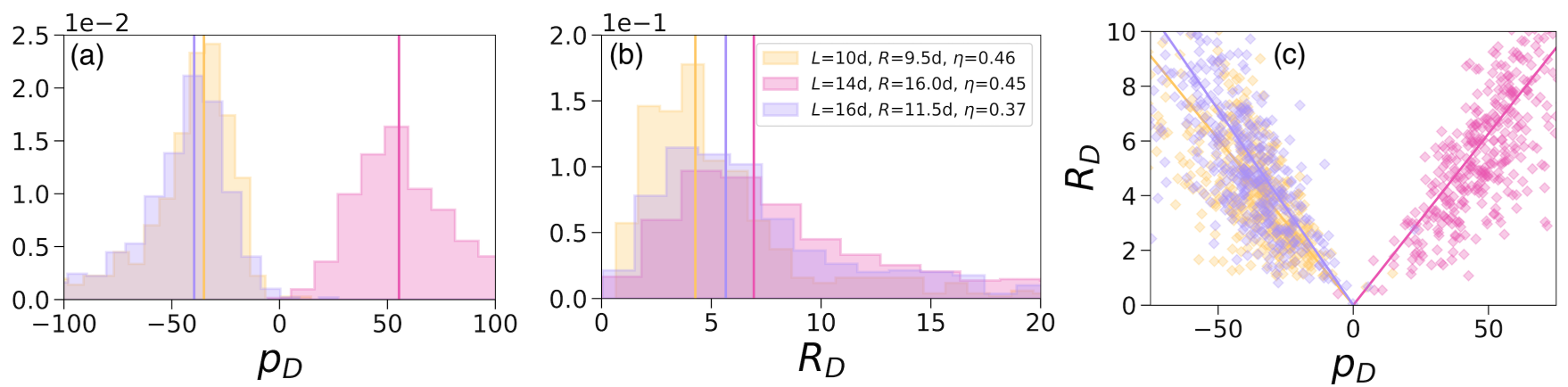

FIG. 5. Probability distribution function of (a) pitches $p_{D}$ and (b) radii $R_{D}$ of the helicoidal dynamics of HCSs of different length $L$, curvature $R$ and packing fraction $\eta$. Positive and negative pitch indicate right and left handedness, respectively. The vertical lines indicate the pitch of the corresponding pseudohelices as predicted by $\mathcal{P}=p$ and $\mathcal{R}=|p| \tan \theta_{0} / 2 \pi$. (c) Radius $R_{D}$ versus the pitch $p_{D}$ of the helicoidal dynamics. The lines correspond to the linear relationship $\mathcal{R}=\mathcal{P} \tan \theta_{0} / 2 \pi$.

[42], an unsupervised manifold learning algorithm that extrapolates from a data set the underlying non-linear manifold from which it is sampled - i.e. a topological space of dimensionality lower or equal to the data set's dimensionality —, has been employed to unveil the low-dimensional coarse-graining of high-dimensional trajectories [43-46]. Here, we exploit DMs to distinguish dynamically distinct parts of a single-particle trajectory consisting of data points $\left\{\mathbf{r}_{i}=\left(x_{i}, y_{i}, z_{i}\right)\right\}$ in 3D. Applying DMs to such a data set enables us to find the true dimensionality, say $d$, of the trajectory and a reparameterization $\left\{\mathbf{r}_{i} \rightarrow \boldsymbol{\Xi}_{i}=\left(\Psi_{2, i}, \Psi_{3, i}, \cdots\right)\right\}$ which clusters points that are close along the $d$-dimensional manifold, thereby getting rid of the variance of the data orthogonal to the manifold [30].

Exemplarily, we consider the trajectory $\left\{\mathbf{r}_{i}=\right.$ $\left.\left(x_{i}, y_{i}, z_{i}\right)\right\}$ containing the coordinates of a HCS in the 3D space as shown in FIG. 4 (a). Applying DMs to this trajectory, we find a dimensionality between 1 and 2, which we attribute to the multiple 1-dimensional helicoidal parts composing the full trajectory. We hence consider only the two leading components of the reparameterization, and represent the trajectory in the 2-dimensional space $\left\{\boldsymbol{\Xi}_{i}=\left(\Psi_{2, i}, \Psi_{3, i}\right)\right\}$ (FIG. 4 (b)). The non-linear transformation $\mathbf{r}_{i} \rightarrow \boldsymbol{\Xi}_{i}$ does not provide any physical insight on the trajectory. However, points $\boldsymbol{\Xi}_{i}$ that are close in the reparametrized space are close along the helicoidal manifolds in the 3D Euclidean space. Hence, a Hierarchical Agglomerative Clustering (HAC) [30, 47] on the reparametrized trajectory $\left\{\boldsymbol{\Xi}_{i}\right\}$ yields clusters of points that are close along these manifolds (FIG. 4 (c)). Remarkably, the respective clusters correspond to the helicoidal segments (FIG. 4 (d)) composing the trajectory in real space. Subsequently, the various helicoidal segments of a trajectory in real space can be fitted with the equation of a helix to obtain the radius $R_{D}$ and pitch $p_{D}$ of the helicoidal dynamics. Applying this procedure to particle trajectories of various $N_{T B}$ phases of HCSs with a particle length, particle curvature, and packing fraction $(L, R, \eta)=(10 d, 9.5 d, 0.46),(14 d, 16 d, 0.45)$, and $(16 d, 11 d, 0.37)$ we find probability distributions of pitches $p_{D}$ and radii $R_{D}$ of the helicoidal dynamics as shown in FIG. 5.

In order to unveil the relationship between the structural and dynamical properties of the $N_{T B}$ phase, we introduce the concept of pseudohelices. As already discussed, curved rods in an $N_{T B}$ phase diffuse preferentially in the direction parallel to the nematic director field $\hat{\mathbf{n}}(z)$, which not only enforces a chirality of the singleparticle dynamics, but also implies that the average particle trajectories are tangent to $\hat{\mathbf{n}}(z)$. Intriguingly, the manifolds tangent to the nematic director field $\hat{\mathbf{n}}(z)$ of an $N_{T B}$ phase with pitch $p$ and conical angle $\theta_{0}$ are helices with a pitch $\mathcal{P}=p$ and radius $\mathcal{R}=|p| \tan \theta_{0} / 2 \pi$, but with different centers [30]. We refer to these helices as pseudohelices generalising the concept of pseudolayers, well-established for cholesteric phases, i.e. planes to which particles are locally tangent [48-51]. For each $N_{T B}$ phase we calculate the pitch $\mathcal{P}$ and radius $\mathcal{R}$ of the pseudohelices, and denote them as vertical lines in FIG. 5. Remarkably, the measured probability distributions of pitches $p_{D}$ and radii $R_{D}$ of the helicoidal trajectories are clearly peaked at these values, confirming that pseudohelices correspond to the average particle trajectory as curved rods follow the chiral twist of the director $\hat{\mathbf{n}}(z)$ in their diffusion, thereby connecting the structural and dynamical properties of the $N_{T B}$ phase. Combining the expressions for the pitch $\mathcal{P}$ and radius $\mathcal{R}$ of a pseudohelix, we find the linear relationship $\mathcal{R}=\mathcal{P} \tan \theta_{0} / 2 \pi$, which describes well our simulation data in FIG. 5c.

In conclusion, we investigated the macroscopic transport properties of the constituent particles in $N_{T B}$ phases using a novel DMC method that accounts for the rototranslational coupling in the diffusion of biaxial particles. Our study shows that the long-time diffusion in the direction parallel to the global nematic director slows down with increasing density but also upon increasing the conical angle. In addition, we studied the dynamics of single curved rods to gain insight in the macroscopic transport properties on a microscopic level. We find that curved 
rods diffuse along non-linear 1D manifolds embedded in the 3D Euclidean space. Using a non-linear dimensionality reduction method, we identify these manifolds with pseudohelices with the same pitch and handedness as the macroscopic $N_{T B}$ phase, thereby linking its structural and dynamical properties and demonstrating that the macroscopic chiral symmetry breaking enforces a chiral helicoidal dynamics at the single-particle level. Our newly devised DMC method to simulate Brownian particles with a nontrivial roto-translational coupling and the dimensionality reduction method to project particle trajectories onto a low-dimensional manifold can be generalized to study the dynamics of arbitrarily shaped particles on various manifolds, e.g. the long-time diffusion in splay-bend nematics [18]. Furthermore, our study provides an insight into the macroscopic transport properties and reveals a link between the structural and dynamical properties in $N_{T B}$ phases, which are both relevant for applications that rely on the type and speed of the macroscopic diffusion, e.g. fast-switching opto-electronic $N_{T B}$-based devices [52, 53], separation of enantiomers in chiral $N_{T B}$ phases $[54,55]$, etc.

\section{ACKNOWLEDGMENTS}

The authors gratefully thank Emanuele Boattini for insightful discussions on Diffusion Maps and unsupervised learning methods. This project has received funding from the European Union's Horizon 2020 research and innovation programme under grant agreement No 676045 .
[1] R. J. Bushby and O. R. Lozman, Current Opinion in Colloid \& Interface Science 7, 343 (2002).

[2] K. Ohta, K. Hatsusaka, M. Sugibayashi, M. Ariyoshi, K. Ban, F. Maeda, R. Naito, K. Nishizawa, A. M. V. de Craats, and J. M. Warman, Molecular Crystals and Liquid Crystals 397, 25 (2003).

[3] S. Kumar, Chem. Soc. Rev. 35, 83 (2006).

[4] S. M. Kelly and M. O'Neill, in Handbook of Advanced Electronic and Photonic Materials and Devices, edited by H. S. Nalwa (Academic Press, Burlington, 2001) pp. $1-66$.

[5] S. Laschat, A. Baro, N. Steinke, F. Giesselmann, C. Hägele, G. Scalia, R. Judele, E. Kapatsina, S. Sauer, A. Schreivogel, and M. Tosoni, Angewandte Chemie International Edition 46, 4832 (2007).

[6] M. Šepelj, A. Lesac, U. Baumeister, S. Diele, H. L. Nguyen, and D. W. Bruce, J. Mater. Chem. 17, 1154 (2007).

[7] V. P. Panov, M. Nagaraj, J. K. Vij, Y. P. Panarin, A. Kohlmeier, M. G. Tamba, R. A. Lewis, and G. H. Mehl, Phys. Rev. Lett. 105, 167801 (2010).

[8] M. Cestari et al., Phys. Rev. E 84, 031704 (2011).

[9] P. A. Henderson and C. T. Imrie, Liquid Crystals 38, 1407 (2011).

[10] K. Adlem, M. Čopič, G. R. Luckhurst, A. Mertelj, O. Parri, R. M. Richardson, B. D. Snow, B. A. Timimi, R. P. Tuffin, and D. Wilkes, Phys. Rev. E 88, 022503 (2013).

[11] V. Borshch, Y.-K. Kim, J. Xiang, M. Gao, A. Jákli, V. P. Panov, J. K. Vij, C. T. Imrie, M. G. Tamba, G. H. Mehl, and O. D. Lavrentovich, Nature Communications 4, 2635 (2013).

[12] N. Sebastián, D. O. López, B. Robles-Hernández, M. R. de la Fuente, J. Salud, M. A. Pérez-Jubindo, D. A. Dunmur, G. R. Luckhurst, and D. J. B. Jackson, Phys. Chem. Chem. Phys. 16, 21391 (2014).

[13] R. J. Mandle, E. J. Davis, S. A. Lobato, C. A. Vol, S. J. Cowling, and J. W. Goodby, Phys. Chem. Chem. Phys. 16, 6907 (2014).

[14] R. J. Mandle, E. J. Davis, C. T. Archbold, S. J. Cowling, and J. W. Goodby, J. Mater. Chem. C 2, 556 (2014).

[15] R. J. Mandle, E. J. Davis, C. T. Archbold, C. C. A.
Voll, J. L. Andrews, S. J. Cowling, and J. W. Goodby, Chemistry - A European Journal 21, 8158 (2015).

[16] E. Gorecka, N. Vaupotič, A. Zep, D. Pociecha, J. Yoshioka, J. Yamamoto, and H. Takezoe, Angewandte Chemie International Edition 54, 10155 (2015).

[17] D. Chen, M. Nakata, R. Shao, M. R. Tuchband, M. Shuai, U. Baumeister, W. Weissflog, D. M. Walba, M. A. Glaser, J. E. Maclennan, and N. A. Clark, Phys. Rev. E 89, 022506 (2014).

[18] M. Chiappini, T. Drwenski, R. van Roij, and M. Dijkstra, Phys. Rev. Lett. 123, 068001 (2019).

[19] R. B. Meyer, in Molecular Fluids, edited by R. Balian and G. Weil (Gordon and Breach, New York, 1976) pp. 271-343.

[20] I. Dozov, EPL (Europhysics Letters) 56, 247 (2001).

[21] R. Memmer, Liquid Crystals 29, 483 (2002).

[22] D. Chen, J. H. Porada, J. B. Hooper, A. Klittnick, Y. Shen, M. R. Tuchband, E. Korblova, D. Bedrov, D. M. Walba, M. A. Glaser, J. E. Maclennan, and N. A. Clark, Proceedings of the National Academy of Sciences 110, 15931 (2013).

[23] C. Greco and A. Ferrarini, Phys. Rev. Lett. 115, 147801 (2015).

[24] A. Patti and A. Cuetos, Phys. Rev. E 86, 011403 (2012).

[25] A. Cuetos and A. Patti, Phys. Rev. E 92, 022302 (2015).

[26] D. Corbett, A. Cuetos, M. Dennison, and A. Patti, Phys. Chem. Chem. Phys. 20, 15118 (2018).

[27] M. Chiappini, E. Grelet, and M. Dijkstra, Phys. Rev. Lett. 124, 087801 (2020).

[28] A. F. D. García, A. Cuetos, and A. Patti, Phys. Rev. E 102, 013302 (2020).

[29] A. Cuetos and A. Patti, Phys. Rev. E 101, 052702 (2020).

[30] See Supplemental Material at http://... for an a posteriori demonstration of the validity of our generalisation of DMC to biaxial particles, details on the self-diffusion of HCSs, a diagram of the anisotropy of the diffusion of hard curved spherocylinders as a function of packing fraction, details on the DMs method, details on the clustering of the trajectories in the low-dimensional space, and a derivation of the pitch and radius of pseudohelices. Refs. [33, 47, 56-58].

[31] C. A, E. M. Rafael, D. Corbett, and A. Patti, Soft Matter 
15, $1922(2019)$.

[32] G. Pajak, L. Longa, and A. Chrzanowska, PNAS 115, E10303 (2018).

[33] J. G. de la Torre, G. del Rio Echenique, and A. Ortega, The Journal of Physical Chemistry B 111, 955 (2007).

[34] D. Frezzato, C. Zannoni, and G. J. Moro, The Journal of Chemical Physics 125, 104903 (2006).

[35] A. Chakrabarty, A. Konya, F. Wang, J. V. Selinger, K. Sun, and Q.-H. Wei, Phys. Rev. Lett. 111, 160603 (2013).

[36] A. Chakrabarty, A. Konya, F. Wang, J. V. Selinger, K. Sun, and Q.-H. Wei, Langmuir 30, 13844 (2014).

[37] A. Chakrabarty, F. Wang, K. Sun, and Q.-H. Wei, Soft Matter 12, 4318 (2016).

[38] C. Hoell and H. Löwen, The Journal of Chemical Physics 144, 174901 (2016).

[39] H. Löwen, Phys. Rev. E 59, 1989 (1999).

[40] M. P. Lettinga, E. Barry, and Z. Dogic, Europhysics Letters (EPL) 71, 692 (2005).

[41] K. Champion, B. Lusch, J. N. Kutz, and S. L. Brunton, Proceedings of the National Academy of Sciences 116, 22445 (2019).

[42] R. R. Coifman, S. Lafon, A. B. Lee, M. Maggioni, B. Nadler, F. Warner, and S. W. Zucker, Proceedings of the National Academy of Sciences 102, 7426 (2005).

[43] B. E. Sonday, M. Haataja, and I. G. Kevrekidis, Phys. Rev. E 80, 031102 (2009).

[44] A. L. Ferguson, A. Panagiotopoulos, P. G. Debenedetti, and I. G. Kevrekidis, Proceedings of the National Academy of Sciences 107, 13597 (2010).

[45] A. L. Ferguson, S. Zhang, I. D., A. Z. Panagiotopoulos, P. G. Debenedetti, and A. J. Link, Biophysical Journal
99, 3056 (2010).

[46] A. L. Ferguson, A. Panagiotopoulos, P. G. Debenedetti, and I. G. Kevrekidis, The Journal of Chemical Physics 134, 135103 (2011).

[47] L. Rokach and O. Maimon, "Clustering methods," in Data Mining and Knowledge Discovery Handbook, edited by O. Maimon and L. Rokach (Springer US, Boston, MA, 2005) pp. 321-352.

[48] W. J. A. Gossens, Molecular Crystals and Liquid Crystals 12, 237 (1971).

[49] A. Wulf, The Journal of Chemical Physics 59, 1487 (1973).

[50] E. H. Korte, Molecular Crystals and Liquid Crystals 44, 151 (1978).

[51] G. S. Chilaya and L. N. Lisetski, Molecular Crystals and Liquid Crystals 140, 243 (1986).

[52] J. Etxebarria and M. B. Ros, Journal of Materials Chemistry 18, 2919 (2008).

[53] A. Jákli, O. D. Lavrentovich, and J. V. Selinger, Reviews of Modern Physics 90, 045004 (2018).

[54] D. Frezzato, G. J. Moro, and C. Zannoni, The Journal of chemical physics 122, 164904 (2005).

[55] D. Frezzato, C. Zannoni, and G. J. Moro, The Journal of chemical physics 125, 104903 (2006).

[56] H. Brenner, Journal of Colloid and Interface Science 23, 407 (1967).

[57] R. R. Coifman, Y. Shkolnisky, F. J. Sigworth, and A. Singer, IEEE Transactions on Image Processing 17, 1891 (2008).

[58] S. Salvador and P. Chan, in 16th IEEE International Conference on Tools with Artificial Intelligence (2004) pp. 576-584. 